\title{
Grupo terapêutico fonoaudiológico de linguagem: revisão de literatura integrativa
}

\section{Speech therapy language therapeutic group: an integrative literature review}

\section{Terapia del lenguaje en grupo: revisión integradora de la literatura}

\author{
Cinthia Procópio da Silva* \\ Regina Tangerino de Souza Jacob* \\ Thais Freire* (D) \\ Patrícia Abreu Pinheiro Crenitte* \\ Aline Roberta Aceituno da Costa* (D)
}

\section{Resumo}

Objetivo: investigar os efeitos do grupo terapêutico em linguagem descritos em estudos brasileiros. Estratégia de pesquisa: a pesquisa foi realizada nas bases eletrônicas Biblioteca Virtual em Saúde (BVS - Bireme) e SciELO. Critérios de seleção: artigos de intervenção em linguagem, realizados em grupo; artigos disponíveis na íntegra e em língua portuguesa; população-alvo abrangendo pré-escolares, escolares, adultos e idosos; artigos publicados no período de 1980 a janeiro 2018; artigos de terapia direta. Critérios de exclusão: artigos de revisão de literatura; artigos repetidos entre as bases eletrônicas; artigos envolvendo terapias individuais ou terapia indireta. A partir dos critérios de inclusão e exclusão, dez estudos foram considerados e analisados. Foram realizadas análises descritivas dos estudos, considerando: ano de publicação, objetivo do estudo, amostragem, método de avaliação pré- e pós-intervenção, estruturação das sessões terapêuticas, referencial teórico e principais resultados. Resultados: verificou-se diversidade de objetivos terapêuticos, de estruturação dos atendimentos e faixa etária dos participantes, $40 \%$ dos autores descreveram o referencial teórico. Os instrumentos de avaliação foram relatados em $70 \%$ dos estudos. Portanto, constatou-se escassez de estudos que exploram os efeitos da terapia coletiva de linguagem.

* Faculdade de Odontologia de Bauru - Universidade de São Paulo, Bauru, São Paulo, Brasil

Contribuição dos autores:

CPS e TF: participaram da coleta dos dados, análise, interpretação dos dados e redação do artigo.

RTSJ, PAPC e ARAC: participaram na condição de orientadoras, na idealização do estudo, análise, interpretação dos dados e redação do artigo.

E-mail para correspondência: Cinthia Procópio da Silva - cinthia.procopio.s@gmail.com Recebido: $18 / 07 / 2020$

Aprovado: 28/10/2020 
Conclusão: destaca-se a necessidade de maiores investimentos na realização e estruturação das terapias fonoaudiológicas em grupo e a divulgação dessas informações.

Palavras-chave: Terapia da Linguage;. Prática de grupo; Revisão; Literatura de revisão como assunto.

\section{Abstract}

Purpose: this study aims to investigate the effects of the Group therapy in language described in Brazilian studies. Research strategies: the search was performed in the electronic databases Virtual Health Library - (VHL Regional Portal) and SciELO. Inclusion criteria: group intervention studies in language; studies available in full and in Portuguese; target population comprising preschoolers, schoolchildren, adults and the elderly; studies published from 1980 to January 2018; direct therapy studies. Exclusion criteria: literature review studies; studies duplicated between electronic databases studies involving individual therapies or indirect therapy. As a result, ten studies were incorporated into the review. The studies' descriptive analysis was performed based on the following items: year of publication, study's objective, sampling, pre- and post-intervention evaluation method, session's structure, theoretical framework and main results. Results: the objectives, the profile of the participants and the structure of the services were diversified. The theoretical framework for group formation was described in $40 \%$ studies, $70 \%$ described the evaluation instruments. There was a scarcity of studies exploring the effects of speech and language therapy. Conclusion: there is a need for greater investments in the development and structuring of speech and language therapy in group and the dissemination of this information.

Keywords: Language Therapy; Group Practice; Review; Review Literature as Topic.

\section{Resumen}

Objetivo: investigar los efectos del grupo terapéutico en el lenguaje descrito en estudios brasileños. Estrategia de investigación: la investigación fue hecha utilizando la Biblioteca Virtual en Salud (BVS Bireme) y las bases de datos electrónicas SciELO. Criterios de selección: artículos de intervención del lenguaje, realizados en grupo; artículos disponibles en su totalidad y en portugués; población objetivo que comprende preescolares, escolares, adultos y ancianos; artículos publicados desde 1980 hasta enero de 2018; artículos de terapia directa. Criterios de exclusión: artículos de revisión de literatura; artículos repetidos entre bases electrónicas; artículos relacionados con terapias individuales o terapia indirecta. A partir de los criterios de inclusión y exclusión, se consideraron y analizaron diez estudios. Se realizaron análisis descriptivos de los estudios, considerando: año de publicación, objetivo del estudio, muestreo, método de evaluación antes y después de la intervención, estructuración de sesiones terapéuticas, referencial teórico y resultados principales. Resultados: hubo una diversidad de objetivos terapéuticos, de como estructurar la atención y el grupo de edad de los participantes. $40 \%$ describieron el referencial teórico e 70\% los instrumentos de evaluación. Por lo tanto, hubo una escasez de estudios que exploren los efectos de la terapia colectiva del lenguaje. Conclusión: existe la necesidad de mayores inversiones en la realización y estructuración de la terapia del lenguaje em grupo y la difusión de esta información.

Palabras clave: Terapia del Lenguaje; Práctica de Grupo; Revisión; Literatura de Revisión como Asunto. 


\section{Introdução}

Desde o surgimento da Fonoaudiologia os atendimentos individuais são priorizados como opção terapêutica. Porém, desde a década de 1980 há iniciativas voltadas para o atendimento coletivo, principalmente em instituições de saúde pública. As primeiras conduzidas em grupos surgiram a partir da necessidade de redução do tempo de espera pelo serviço em razão do aumento na demanda de pacientes $^{1}$.

Destaca-se que a formação dos grupos geralmente considera o perfil de homogeneidade ou heterogeneidade dos indivíduos. Nos homogêneos, leva-se em consideração a faixa etária e/ ou patologia semelhante; a intenção é agrupar os participantes com características relacionadas. Nos heterogêneos, por sua vez, os indivíduos podem apresentar discrepâncias diagnósticas e etárias. Além dessas características, existem estudos que relatam os referenciais teóricos que sustentam a elaboração e condução dos grupos ${ }^{2}$.

O referencial teórico adotado na avaliação da terapia de linguagem relaciona-se com a concepção teórica adotada pelo avaliador, portanto há variadas práticas e perspectivas teóricas ${ }^{2}$. Os profissionais que optam pelo atendimento grupal, em sua maioria, adotam uma perspectiva sócio-interacionista, pois acreditam que a linguagem se estabelece e evidencia-se na interação social e na troca de diálogo entre interlocutores, em um ambiente onde o fenômeno da linguagem está intrinsicamente presente $^{2,3}$.

Podem ser citados como referenciais teóricos utilizados o sócio-interacionista e interacionista, psicologia positiva, histórico-cultural, além de autores que derivam desses princípios. Há ainda, autores que não esclarecem a teoria em que se baseiam ${ }^{4}$.

Atualmente, o atendimento coletivo é visto por muitos profissionais como uma abordagem benéfica, pois pode promover redução de custos, estreitar a relação entre os participantes, propiciar intercâmbio de experiências, construção conjunta do conhecimento entre os indivíduos, respeito das diferenças, apoio na vivência das outras pessoas, entre outros pontos positivos. Em um estudo comparativo foram analisados os custos de quatro tipos de intervenções e constatou-se que a terapia indireta em grupo foi a menos onerosa, seguida da terapia direta em grupo, maiores gastos foram evidenciados nas terapias individuais ${ }^{5}$.

Em relação aos métodos de avaliação de linguagem, a literatura descreve que a maioria dos profissionais realiza avaliações individuais antes de iniciar os atendimentos grupais, apoiando-se nos instrumentos da avaliação individual e uma pequena parcela não realiza avaliação específica fonoaudiológica ${ }^{6}$. Há carência de instrumentos para avaliação coletiva da linguagem, o que pode justificar a preferência por procedimentos individuais. Ainda, ocorrem críticas aos modelos individuais, sobretudo quando conduzidos em crianças pequenas; os autores alegam timidez e insegurança das crianças diante de um avaliador desconhecido o que pode interferir nos resultados observados. Partindo desse pressuposto, há iniciativas que utilizam o próprio grupo fonoaudiológico para realização da avaliação da linguagem dos participantes ${ }^{3}$.

As relações terapêuticas têm sido estudadas na literatura, o fonoaudiólogo desempenha variados papéis no atendimento em grupo: coordenador, participante- coordenador, mediador, agente, interlocutor ativo, demonstrando ser o detentor do saber ${ }^{2}$.

Os atendimentos grupais estão sendo desenvolvidos com crianças, adolescentes, adultos, idosos, pais e professores, nas mais diversas áreas de atuação da Fonoaudiologia, e os relacionados à Linguagem representam o maior número de publicações ${ }^{4,7}$.

\section{Objetivo}

O objetivo do presente estudo foi realizar um levantamento de dados para verificar os efeitos do grupo terapêutico em linguagem, descritos em estudos brasileiros.

\section{Métodos}

\section{Estratégia de pesquisa}

Trata-se de estudo de revisão integrativa de artigos nacionais abordando a temática: grupos terapêuticos fonoaudiológicos na área da linguagem. O processo de busca foi realizado por dois pesquisadores, que seguiram a mesma metodologia: busca independente dos artigos, análise às cegas, leitura inicial do título e do resumo dos artigos a partir das combinações entre os descritores e palavras chaves utilizadas e os critérios de inclusão e exclusão. 
Quando o título e o resumo não foram esclarecedores, o artigo foi lido na íntegra. Em seguida, os artigos selecionados por cada pesquisador que atendiam à proposta da revisão foram comparados e analisados criticamente pelos examinadores e os critérios de inclusão e exclusão foram aplicados. As discordâncias que por ventura ocorreram foram resolvidas por consenso.

Foram acessadas as bases de dados eletrônicas: Biblioteca Virtual em Saúde: BVS - (Bireme) e SciELO, ambas foram escolhidas por serem bases com acesso aberto à informação científica e técnica em saúde. A Biblioteca Virtual em Saúde (BVS) abrange a América Latina e Caribe (AL\&C), sendo que as fontes de informação do portal são compostas dos dados produzidos pela Rede BVS, como LILACS e Medline; a Scientific Electronic Library Online - SciELO é uma biblioteca eletrônica que abrange uma coleção selecionada de periódicos científicos brasileiros. Foram incorporados à pesquisa apenas os artigos disponíveis na íntegra, acessados por meio da Rede Privada Virtual (VPN) de uma instituição de ensino superior pública. Para rastrear os artigos de interesse da pesquisa, foram utilizadas como estratégias de busca as combinações entre termos cadastrados no Descritores em Ciências da Saúde (DeCS) e palavras-chave em português, descritas na Tabela 1.

Tabela 1. Estratégia de busca utilizada nas bases de dados

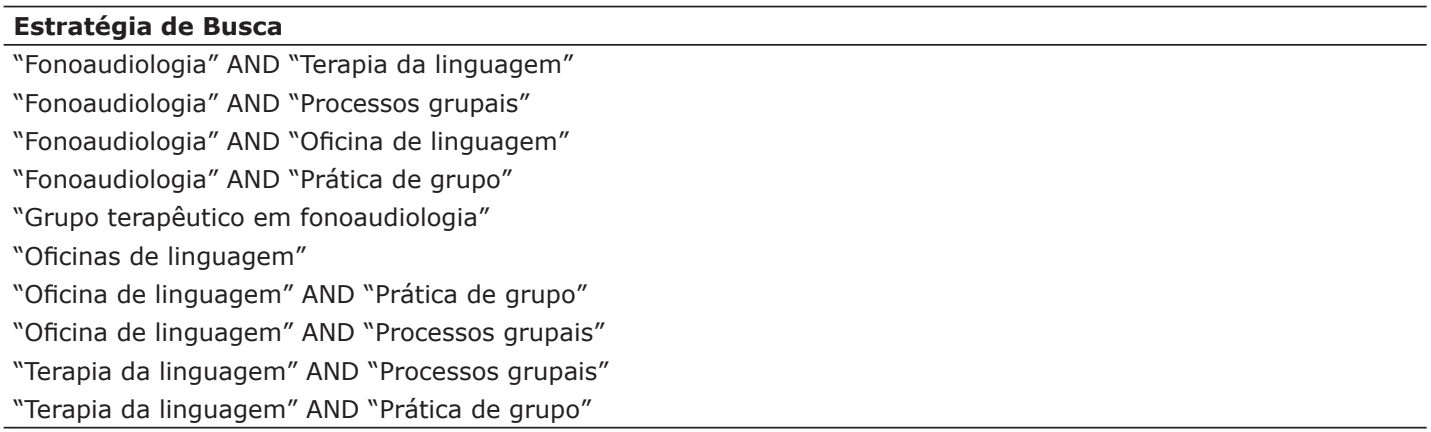

\section{Critérios de seleção}

Os artigos foram selecionados obedecendo aos critérios de inclusão e exclusão. Adotou-se como critério de inclusão: artigos de intervenção em linguagem, realizados em grupo; artigos disponíveis na íntegra e em língua portuguesa; população-alvo abrangendo pré-escolares, escolares, adultos e idosos; artigos publicados no período de 1980 a janeiro de 2018; artigos de terapia direta, onde as técnicas e abordagens são direcionadas ao indivíduo. Os critérios de exclusão foram: artigos de revisão de literatura; artigos repetidos entre as bases eletrônicas; artigos envolvendo terapias individuais ou terapia indireta, na qual são realizadas orientações ao indivíduo e familiares.

Foram encontrados 179 resultados nas bases de dados considerando as estratégias de busca (Figura 1). Destes, apenas 49 relacionavam-se à área de linguagem e à fonoaudiologia e, aplicando-se os critérios de inclusão e exclusão, dez artigos no total foram considerados para esta revisão (Tabela 2). 

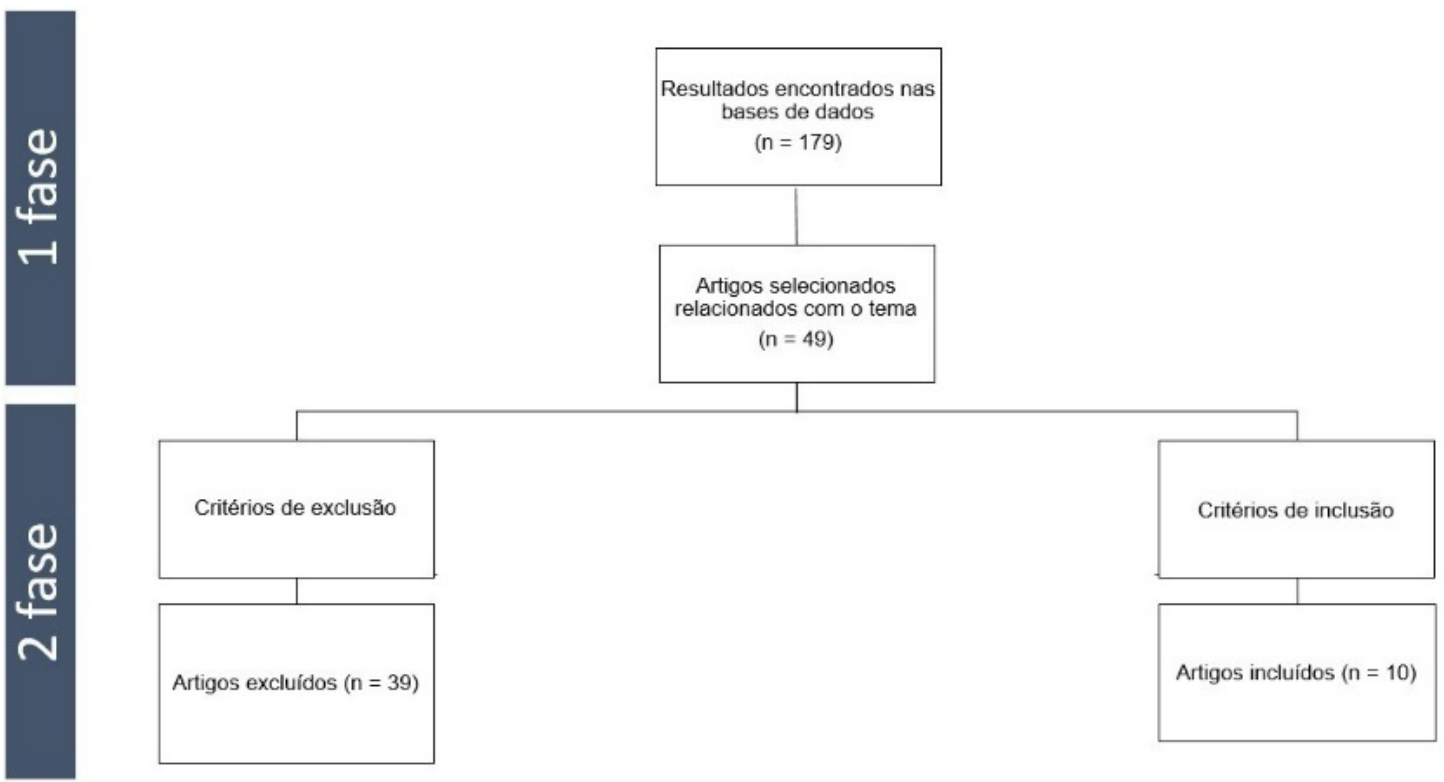

Figura 1. Artigos selecionados adotando-se os critérios de inclusão e exclusão

Tabela 2. Artigos publicados por ano

\begin{tabular}{|c|c|c|}
\hline Autor & Nome do artigo & Ano de publicação \\
\hline Cardoso C, Fernandes FDM. & $\begin{array}{c}\text { Relação entre os aspectos sócio cognitivos e perfil } \\
\text { funcional da comunicação em um grupo de adolescentes } \\
\text { do espectro autístico. }\end{array}$ & 2006 \\
\hline Freitas AP, Castro GS. & $\begin{array}{l}\text { A constituição de processos dialógicos em um grupo de } \\
\text { jovens com deficiência mental. }\end{array}$ & 2006 \\
\hline $\begin{array}{c}\text { Fernandes FD, Cardoso C, Sassi } \\
\text { FC, Amato CLH, Sousa-Morato } \\
\text { PF. }\end{array}$ & $\begin{array}{l}\text { Fonoaudiologia e autismo: resultado de três diferentes } \\
\text { modelos de terapia de linguagem. }\end{array}$ & 2008 \\
\hline Soares AJC, Cárnio MS. & $\begin{array}{c}\text { Consciência fonêmica em escolares antes e após } \\
\text { oficinas de linguagem. }\end{array}$ & 2012 \\
\hline $\begin{array}{c}\text { Cardoso C, Rocha JFL, Moreira } \\
\text { CS, Pinto AL. }\end{array}$ & $\begin{array}{c}\text { Desempenho sócio-cognitivo e diferentes situações } \\
\text { comunicativas em grupos de crianças com diagnósticos } \\
\text { distintos. }\end{array}$ & 2012 \\
\hline Lourenço RCC, Massi G, Lima RR. & $\begin{array}{l}\text { Trabalho com a linguagem e envelhecimento: uma } \\
\text { busca por ressignificações de histórias de vida. }\end{array}$ & 2014 \\
\hline $\begin{array}{l}\text { Guarinello AC, Massi G, } \\
\text { Berberian AP, Cláudio DP, Festa } \\
\text { PSV, Carvalho HAS. }\end{array}$ & $\begin{array}{l}\text { A retextualização como prática nas terapias } \\
\text { fonoaudiológicas com sujeitos surdos. }\end{array}$ & 2014 \\
\hline Santana AP, Signor RCF. & $\begin{array}{l}\text { Grupo para sujeitos com queixas de dificuldades de } \\
\text { leitura e escrita: aspectos teórico-metodológicos. }\end{array}$ & 2015 \\
\hline $\begin{array}{l}\text { Sales NJ, Chun RYS, Santos FFD, } \\
\text { Sampaio ATS, Noronha PSV, } \\
\text { Gonçalves RSS; et al. }\end{array}$ & $\begin{array}{l}\text { Ações de graduandos de Fonoaudiologia em Centro de } \\
\text { Referência em Educação Especial do Nordeste. }\end{array}$ & 2015 \\
\hline Zerbeto AB, Batista CG. & $\begin{array}{l}\text { Abordagem grupal para avaliação de alterações de } \\
\text { linguagem em crianças pequenas. }\end{array}$ & 2016 \\
\hline
\end{tabular}




\section{Revisão da Literatura}

Para apresentação dos dados, os artigos foram descritos em ordem cronológica crescente, destacando os seguintes itens: ano de publicação, objetivo do estudo, amostragem, método de avaliação pré- e pós- intervenção, estruturação da sessão de terapia, referencial teórico utilizado e principais resultados.

Do ano de 2006, foram encontrados dois artigos pertinentes à revisão. O primeiro estudo analisou a evolução do perfil funcional da comunicação e do desempenho sócio-cognitivo de cinco adolescentes com transtorno do espectro autista (TEA), em três situações comunicativas diferentes: em terapia de linguagem individual, em grupo com coordenador, e criança em grupo sem coordenador, salientando que o coordenador do grupo não é o terapeuta de linguagem. Gravações em vídeo foram utilizadas como método de avaliação, na primeira e última sessão de terapia, as quais tiveram duração de 30 minutos cada. As filmagens serviram como base para analisar o perfil funcional da comunicação, utilizando os critérios propostos por Fernandes (2004), e para a análise dos aspectos sócio-cognitivos, foram utilizados os critérios propostos por Molini (2001). Não foi relatado como as sessões terapêuticas foram estruturadas ou o referencial teórico para a formação do grupo. Os autores concluíram que existe relação entre a evolução do desempenho sócio-cognitivo e o perfil funcional da comunicação em todos os grupos de terapia ${ }^{8}$.

No segundo estudo de 2006, o objetivo foi promover e analisar as interações dialógicas em grupo. Participaram do estudo quatro jovens com deficiência intelectual. Não foi descrito como os participantes seriam avaliados antes e após as sessões de terapia. O grupo foi atendido semanalmente por uma fonoaudióloga, durante o período de quatro meses. O referencial teórico para formação do grupo foi o sócio-histórico. O estudo apontou que as terapias em grupo favorecem o aumento das intenções comunicativas, pois recriam um ambiente mais propício ao diálogo e ainda, permitem a troca de experiências entre jovens com deficiência?.

Em 2008, um estudo buscou analisar diferenças no desempenho comunicativo e sócio-cognitivo de crianças e adolescentes com autismo, atendidos em três diferentes situações: atendimento individual, em duplas ou atendimento conjunto com as mães. Participaram do estudo 36 indivíduos, avaliados por meio de filmagens na primeira e última sessão terapêutica. Todas as filmagens foram transcritas para a análise dos aspectos comunicativos e sócio-cognitivos. Os atendimentos em grupo foram realizados em 20 sessões, não houve descrição da duração dos encontros e de referenciais teóricos. Dentre os três tipos de atendimentos, os realizados em duplas produziram os melhores resultados no número de atos comunicativos expressos por minuto e aumento na proporção da interatividade comunicativa $^{10}$.

Do ano de 2012 dois artigos foram incorporados à revisão. O primeiro estudo verificou o desempenho de 49 escolares em consciência fonêmica antes e após a realização de oficinas de linguagem. Inicialmente, foi realizada a avaliação da consciência fonêmica por meio do teste "Consciência Fonológica - Instrumento de Avaliação Sequencial" (CONFIAS), os escolares passaram por avaliação final idêntica à inicial. As oficinas foram realizadas uma vez por semana, com duração de 50 minutos cada, totalizando cinco encontros para cada grupo. $\mathrm{O}$ referencial teórico utilizado não foi citado no trabalho. Os resultados demonstraram que a média de acertos no teste foi maior após a realização das oficinas de linguagem, demonstrando uma melhora do desempenho em consciência fonêmica ${ }^{11}$. O segundo estudo, verificou o desempenho sócio-cognitivo de 30 crianças com diagnósticos inseridos nos distúrbios do espectro do autismo, deficiência mental e deficiência auditiva em duas diferentes situações comunicativas, na situação em grupo e na terapia individual. Para avaliação dos participantes, foram realizadas filmagens da terapia individual e em grupo, e para a análise dos dados foi utilizado o teste elaborado por Molini-Avejonas e Fernandes (2003). Não foi descrito como as sessões foram estruturadas ou o referencial teórico utilizado. O estudo destaca que, com os autistas, as terapias em grupo simulam situações mais próximas da realidade e geram melhor desempenho comunicativo quando comparado com terapias individuais ${ }^{12}$.

Em 2014, um estudo se propôs descrever as vivências de idosos participantes de uma Oficina de Linguagem (OL) que realizavam discussões orais e a escrita das suas histórias pessoais. Foram entrevistados dez idosos de uma Unidade de Saúde de Curitiba/PR, que participavam das oficinas de linguagem semanalmente, com encontros que duravam 90 minutos. $\mathrm{O}$ trabalho da coordenadora da oficina era fundamentado na filosofia de linguagem 
de Bakhtin, filosofia esta que pressupõe conceber a linguagem como um fenômeno sócio-interacional e dialógico. Os idosos foram avaliados qualitativamente por meio de entrevista semi-estruturada. Os autores concluíram pelos relatos coletados que as oficinas de linguagem ajudaram os idosos a (re) significar as suas histórias em relação à velhice e à vida, e a serem inseridos na sociedade ${ }^{13}$.

Também em 2014, foi desenvolvido um projeto com o objetivo de promover a retextualização em terapias fonoaudiológicas em grupo, para promover o desenvolvimento da linguagem escrita. A retextualização é o processo de produção de um novo texto a partir de um texto-base, neste caso em específico, seria a transformação das informações da língua de sinais em textos na língua portuguesa. Participaram oito deficientes auditivos que utilizam Libras e a Língua Portuguesa em sua modalidade escrita. O método de avaliação dos indivíduos não foi descrito no estudo, assim como o referencial teórico empregado. As terapias tinham a duração de duas horas cada encontro e ocorriam semanalmente. Por meio da retextualização em grupo, os deficientes auditivos passaram a refletir sobre a escrita e sua função social, assumindo essa modalidade da linguagem com mais disposição e autoconfiança ${ }^{14}$.

$\mathrm{O}$ primeiro estudo incorporado à revisão do ano de 2015 teve como objetivo apresentar em conformidade à teoria enunciativo-discursiva, os pressupostos teórico-metodológicos que sustentam a terapia fonoaudiológica em grupo voltada ao desenvolvimento de competências em linguagem escrita e analisar sua eficácia. Fizeram parte do estudo cinco adolescentes com queixas de dificuldades de leitura e de escrita. O método de avaliação dos indivíduos não foi mencionado no trabalho. Os atendimentos foram desenvolvidos em sessões fonoaudiológicas semanais que tinham por volta de três horas de duração cada. O referencial teórico aplicado foi o sócio-interacionista, da noção bakhtiniana de gêneros do discurso. De acordo com o estudo, a constituição do grupo proporciona, além das trocas de conhecimentos, um sentimento de união que eleva a autoestima, favorecendo os processos de aprendizagem ${ }^{15}$.

Ainda em 2015, um artigo objetivou descrever ações de fonoaudiologia em um Centro de Referência em Educação Especial - CREESE/ SEED do Estado de Sergipe, junto aos usuários e seus familiares. As intervenções foram realizadas por graduandos do curso de Fonoaudiologia supervisionados por um docente, com atendimentos individuais e grupais, as ações foram gravadas e transcritas, realizadas na seguinte ordem: diagnóstico situacional do CREESE, entrevista com os responsáveis, avaliação fonoaudiológica e devolutiva aos pais, oficinas de linguagem e devolutiva final aos responsáveis da proposta elaborada. Participaram do estudo 27 usuários, 19 meninos $(70 \%)$ e 23 meninas $(85 \%)$ do ensino regular ${ }^{16}$.

Foram realizadas avaliações iniciais nas áreas de linguagem, onde foi utilizado o Teste de Rastreamento de Distúrbios Articulatórios de Fala - TERDAF na análise da linguagem oral e a construção de um texto ou elaborado um ditado de letras e palavras para avaliar a linguagem escrita; na voz, foi utilizada a escala Consensus Auditory-Perceptual Evaluation Voice - CAPE-V; na motricidade orofacial e funções estomatognáticas, foi utilizado o Protocolo de Avaliação Miofuncional Orofacial com Escores - AMIOFE; na audição foi utilizada a Triagem do Processamento Auditivo Central. Tais instrumentos permitiram definir as atividades oferecidas, considerando as dificuldades e potencial dos participantes. Foi realizada também, comparação dos aspectos linguísticos, perceptuais e de interação pré e pós as oficinas de linguagem ${ }^{16}$.

$\mathrm{O}$ atendimento individual serviu como base para que os alunos analisassem os déficits e a capacidade linguístico-cognitiva dos participantes. Os grupos realizaram em média cinco encontros, com duração de 60 minutos e eram constituídos por três usuários e dois mediadores graduandos de fonoaudiologia, totalizando oito grupos. Não foi citado no estudo qual o referencial teórico utilizado, porém salientou-se que para a constituição dos grupos não foi considerada a homogeneidade de hipóteses diagnósticas médica e fonoaudiológica, dessa maneira, todos os participantes foram incluídos ${ }^{16}$.

Considerando a individualidade de cada sujeito, foi observados na intervenção grupal diferentes níveis de ganhos. Foi citada uma maior percepção dos participantes quanto às suas dificuldades e potencialidades, diminuição da tensão corporal, favorecimento da interação social, intenção e funcionalidade comunicativa, maior atenção visual ou auditiva, acréscimo na expressividade facial e corporal ou da própria linguagem, entre outros ganhos $^{16}$.

O artigo mais recente foi encontrado do ano de 2016, seu objetivo foi descrever as contribuições 
de uma abordagem terapêutica grupal na avaliação da linguagem de crianças. Participaram do estudo nove crianças, que foram divididas em três grupos, constituídos de três participantes (G1 e G2 - crianças com queixas de alterações de linguagem e com diagnóstico diversificado, G3 - crianças sem queixas de linguagem pertencentes a uma escola pública infantil). Para avaliar a participação de cada criança no grupo terapêutico e as produções verbais e não verbais, foram utilizados vídeos e diário de campo. Foram realizados quatro encontros, com duração entre 30 e 60 minutos cada, com enfoque sócio-interacionista. Os resultados descreveram que a constituição do grupo favoreceu a interação, a identificação do potencial linguístico das crianças, a cooperação entre os participantes, sendo observado quando as crianças com menor dificuldade auxiliaram outras com dificuldades motoras, como também, algumas crianças seguiram exemplos umas das outras, no modo de uso de objetos e na reprodução de falas. Deve ser ressaltado que, por meio de uma avaliação com maior número de sessões, houve um aumento da complexidade nas funções de linguagem utilizadas pelos participantes dos grupos ${ }^{3}$.

Nota-se de fato, uma escassez no número de artigos que abordam a terapia em grupo na Fonoaudiologia, incluindo a área da linguagem. Estudos apontam a necessidade de novos estudos sobre a prática grupal e do desenvolvimento de revisões de literatura sobre o assunto ${ }^{4}$. O fortalecimento da atuação fonoaudiológica na linguagem oral e escrita só acontece quando os resultados são sistematizados, registrados, analisados e divulgados ${ }^{17}$. Deve-se realizar o levantamento dos serviços que oferecem atendimentos grupais na fonoaudiologia e avaliar suas características gerais, assim como outros estudos que efetuaram a caracterização da atenção em outros serviços ${ }^{18}$.

No que diz respeito às avaliações realizadas, pontuaram esse item apenas os estudos que relataram em sua metodologia o uso de instrumentos de avaliação antes e após as sessões de terapia, a fim de reunir dados sobre os estudos que utilizam alguma ferramenta para comprovar seus resultados. Os artigos incluídos na revisão utilizaram instrumentos diversos para as avaliações dos participantes, sendo eles: filmagens, critérios do perfil funcional segundo Fernandes (2003) e (2004), critérios do perfil sócio-cognitivo segundo Molini (2001), teste Molini-Avejonas e Fernandes (2003), Teste Cons- ciência Fonológica - Instrumento de Avaliação Sequencial (CONFIAS), Teste de Rastreamento de Distúrbios Articulatórios de Fala - TERDAF, Escala Consensus Auditory-Perceptual Evaluation Voice - CAPE-V, Protocolo de Avaliação Miofuncional Orofacial com Escores - AMIOFE e Triagem do Processamento Auditivo Central. Para garantir a eficácia dos procedimentos é necessário o planejamento terapêutico e o uso de instrumentos avaliativos, prática que é cada vez mais exigida pelos conselhos de classe ${ }^{17}$.

Um ponto importante a ser discutido é o número de artigos que utilizaram algum instrumento para quantificar os ganhos dos grupos terapêuticos. Nota-se que, dentre os dez estudos analisados, sete descreveram os instrumentos utilizados para constatar a evidência dos resultados obtidos, todos utilizaram o modelo de avaliação individual, não foram citados instrumentos para a avaliação em grupo.

Pesquisas que descrevam questões da dinâmica do trabalho em grupo, que indiquem seus ganhos e limitações, além das lacunas desse modelo de atendimento, auxiliará o fonoaudiólogo a refletir e considerar o atendimento em grupo, além da visão de ser unicamente uma abordagem para suprir a grande demanda de pacientes, mas aplicá-la como uma alternativa de intervenção ${ }^{6}$.

O termo "evidência" deve ser reconhecido, assim como sua função, para que seja compreendida sua aplicabilidade na prática em Fonoaudiologia. Esse termo pode ser compreendido como um conjunto de elementos ou provas que confirmam ou anulam as hipóteses de um determinado estudo, juntamente com a experiência do profissional e as preferências do paciente ${ }^{19}$.

Ainda sobre essa questão, constatou-se que a filmagem das sessões terapêuticas foi utilizada como forma de avaliação em quatro estudos ${ }^{3,8,9,12} \mathrm{e}$ combinada com outros instrumentos. Observa-se que, a filmagem é uma ferramenta que auxilia a análise dos dados e comparação do desempenho dos participantes por mais de um profissional, uma vez que nas terapias em grupo, pode ser difícil a análise das ações dos participantes no momento em que as interações acontecem, pois várias informações e ações ocorrem em um mesmo instante. Apesar de ser uma boa ferramenta, deve ser utilizada em conjunto com outros instrumentos padronizados.

Os trabalhos estruturaram as sessões terapêuticas com características bastante diversas: número 
total de atendimentos, frequência dos atendimentos e tempo de duração. Desse modo, podem-se registrar diferentes modelos de terapia. De acordo com o Manual de Orientação ao Fonoaudiólogo: Balizador de tempo de tratamento em Fonoaudiologia, a duração dos atendimentos depende do distúrbio ou da alteração que o paciente possui; deve-se levar em consideração o desenvolvimento individual durante o tratamento, o grau de comprometimento do indivíduo e da família. Outro aspecto importante a ser considerado é a assiduidade e pontualidade, pois os ganhos terapêuticos não são os mesmos se o paciente apresenta muitas faltas e atrasos. Esse guia foi elaborado pelo Conselho Regional de Fonoaudiologia, nele são descritos os passos para o uso do instrumento balizador de tempo (IBT), por meio desse instrumento é possível ver o tempo médio do tratamento, a frequência semanal e a duração recomendada da consulta/sessão de acordo com a patologia que o paciente apresenta ${ }^{20}$.

A função do fonoaudiólogo em seis dos artigos foi de coordenador e mediador, ou seja, o profissional conduzia e administrava os grupos durante a intervenção. Alguns autores defendem que os próprios pacientes devam cumprir o papel ativo no seu processo terapêutico, referem que o terapeuta deve proporcionar o crescimento e evolução do paciente, fazendo-o obter autoconfiança, independência e integração pessoal, de modo que trace o seu próprio caminho para alcançar seus objetivos ${ }^{21}$.

Em relação à abordagem teórica dos estudos, $30 \%$ descreveram o uso da perspectiva sócio-histórica e $10 \%$ da bakhtiniana. Existe uma diversificada quantidade de princípios teóricos empregados nos atendimentos em grupo na fonoaudiologia e há o predomínio da perspectiva sócio-histórica para a construção dos grupos ${ }^{2}$. Um estudo verificou como e por que foi constituído o grupo de terapia fonoaudiológica com sete fonoaudiólogos e, segundo os entrevistados, o grupo, inicialmente, foi constituído pela demanda ${ }^{6}$. O atendimento em grupo na prática clínica da fonoaudiologia ganhará um norte e sentido a partir do momento em que a explicação teórica das bases que a sustentam seja entendida e empregada pelos profissionais.

A literatura fonoaudiológica demonstra uma inconsistência teórica acerca do referencial teórico empregado nos estudos em grupo, há adoção irrefletida de conceitos de outras áreas. Existe a hipótese que estas incoerências aconteçam devido à falta de articulação dos estudos com os elementos que caracterizam a clínica fonoaudiológica. ${ }^{2}$

\section{Conclusão}

Verifica-se a escassez de estudos que exploram os efeitos da fonoterapia em linguagem quando conduzida em grupos. Atualmente, poucos abordam a temática e, ainda, não existe consenso sobre qual abordagem ou instrumentos de avaliação que possibilitam afirmações sobre eficácia. Aqueles que realizaram avaliações, ainda que individuais, dos participantes dos grupos, demostraram efeitos positivos no desenvolvimento e aprimoramento da linguagem de crianças, adultos e idosos. Neste estudo não foram considerados artigos internacionais, pois a intenção foi verificar a prática fonoaudiológica nacional. Porém, sugere-se que novos estudos sejam realizados visando comparações entre a realidade brasileira e estrangeira para se entender a construção de processos facilitadores do desenvolvimento da linguagem e da subjetividade quando realizada em grupos terapêuticos.

Destaca-se a necessidade de maiores investimentos na realização e estruturação das terapias fonoaudiológicas em grupo, bem como divulgação dessas informações à comunidade. Por este caminho será possível fortalecer a atuação do fonoaudiólogo, principalmente nas instituições públicas de saúde ou educação, nos quais os atendimentos em grupo representam a realidade.

\section{Referências}

1. Corrêa MB. Considerações sobre a terapia de grupo na clínica fonoaudiológica. In: Lier-De Vitto MF. Fonoaudiologia: no sentido da linguagem. São Paulo: Cortez; 1994. p.39-48.

2. Araújo MLB, Freire RMAC. Atendimento fonoaudiológico em grupo. Rev. CEFAC. 2011 abr; 13(2): 362-8.

3. Zerbeto AB, Batista CG. Abordagem grupal para avaliação de alterações de linguagem em crianças pequenas. Ciênc. Saúde Colet. 2016 jan; 21(1): 203-12.

4. Ribeiro VV, Panhoca I, Dassie-Leite AP, Bagarollo MF. Grupo terapêutico em fonoaudiologia: revisão de literatura. Rev. CEFAC. 2012 jun; 14(3): 544-52.

5. Dickson K, Marshall M, Boyle J, McCartney E, O’Hare A, Forbes J. Cost analysis of direct versus indirect and individual versus group modes of manual-based speech-and-language therapy for primary school-age children with primary language impairment. Int. J. Lang. Commun. Disord. 2009 mai; 44(3): 369-81. 
6. Vilela FCA, Ferreira LP. Voz na clínica fonoaudiológica: grupo terapêutico como possibilidade. Distúrb. comun. 2006 ago; 18(2): 235-243.

7. Panhoca I, Penteado RZ. Grupo terapêutico-fonoaudiológico: a construção (conjunta) da linguagem e subjetividade. Pró-Fono. 2003; 15(3): 259-66.

8. Cardoso C, Fernandes FDM. Relação entre os aspectos sócio cognitivos e perfil funcional da comunicação em um grupo de adolescentes do espectro autístico. Pró-Fono. 2006 jan; 18(1): 89-98.

9. Freitas AP, Castro GS. A constituição de processos dialógicos em um grupo de jovens com deficiência mental. Rev. bras. educ. espec. 2006 jan/abr; 12(1): 49-64.

10. Fernandes FD, Cardoso C, Sassi FC, Amato CLH, SousaMorato PF. Fonoaudiologia e autismo: resultado de três diferentes modelos de terapia de linguagem. Pró-Fono. 2008 dez; 20(4): 267-72.

11. Soares AJC, Cárnio MS. Consciência fonêmica em escolares antes e após oficinas de linguagem. J. Soc. Bras. Fonoaudiol. 2012; 24(1): 69-75.

12. Cardoso C, Rocha JFL, Moreira CS, Pinto AL. Desempenho sócio-cognitivo e diferentes situações comunicativas em grupos de crianças com diagnósticos distintos. J. Soc. Bras. Fonoaudiol. 2012 mar; 24(2): 140-4

13. Lourenço RCC, Massi G, Lima RR. Trabalho com a linguagem e envelhecimento: uma busca por ressignificações de histórias de vida. Rev. CEFAC. 2014 abr; 16(2): 672-8.

14. Guarinello AC, Massi G, Berberian AP, Cláudio DP, Festa PSV, Carvalho HAS. A retextualização como prática nas terapias fonoaudiológicas com sujeitos surdos. Rev. CEFAC. 2014 out; 16(5): $1680-90$

15. Santana AP, Signor RCF. Grupo para sujeitos com queixas de dificuldades de leitura e escrita: aspectos teórico-metodológicos. Rev. CEFAC. 2015 dez; 17(6): 1814-26.

16. Sales NJ, Chun RYS, Santos FFD, Sampaio ATS, Noronha PSV, Gonçalves RSS et al. Ações de graduandos de Fonoaudiologia em Centro de Referência em Educação Especial do Nordeste. Distúrb. comun. 2015 set; 27(3): 630-41.

17. Perissinato J, Avila CRB. Avaliação e diagnóstico das linguagens oral e escrita. In: Fernandes DMF, Mendes BCA, organizadores. Tratado de fonoaudiologia. $2^{\mathrm{a}}$ ed. São Paulo: Roca; 2009. p. 275-277.

18. Monlleó IL, Gil-da-Silva-Lopes VL. Anomalias craniofaciais: descrição e avaliação das características gerais da atenção no Sistema Único de Saúde. Cad. Saúde Pública. 2006 maio; 22(5): 913-22.

19. Medrado CS, Sobrinho FPN. Prática Baseada em Evidência (PBE) em Fonoaudiologia. Distúrb. comun. 2016 jun; 28(2): 341-49.

20. CFFA: Conselho Federal de Fonoaudiologia. Balizador de tempo de tratamento em fonoaudiologia. Brasília (DF): CFFA, 2013 [acesso 23 jan 2019]. Disponível em: http://www. fonoaudiologia.org.br/publicacoes/BALIZADOR $\% 20 \mathrm{DE} \% 20$ TEMPO.pdf

21. Souza APR, Crestani AH, Vieira CR, Machado FCM, Pereira LL. O grupo na fonoaudiologia: origens clínicas e na saúde coletiva. Rev. CEFAC. 2011 Fev; 13(1): 140-151. 